\title{
A case of multiple primary malignancies and investigation of family history
}

\author{
XIU-YAN HUANG ${ }^{1 *}$, ZI-LI HUANG ${ }^{2 *}$, JIN HUANG $^{1}$, ZHI-GANG WANG $^{1}$ and QI ZHENG ${ }^{1}$ \\ ${ }^{1}$ Department of General Surgery, The 6th People's Hospital of Shanghai, School of Medicine, Shanghai Jiaotong University, \\ Shanghai 200233; ${ }^{2}$ Department of Radiology, The Central Hospital of Shanghai Xuhui District, Shanghai 200031, P.R. China
}

Received May 8, 2012; Accepted August 6, 2012

DOI: $10.3892 / 01.2012 .885$

\begin{abstract}
The occurrence of multiple primary malignancies (MPM) in one patient is a rare but increasingly frequent event, partly due to medical advances in diagnosis and therapy. A number of theories have been proposed to explain MPM, but none have been proven. A key risk factor appears to be family history. We present the case of a 43-year-old male with multiple cancers who was first admitted to the hospital for an undifferentiated pleomorphic sarcoma/pleomorphic malignant fibrous histiocytoma (pG2T2bN0Mx stage III) of the right scapula in May 2009. The patient underwent three tumor resections in situ due to tumor recurrence. During the period of chemotherapy and radiotherapy, computed tomography (CT) revealed a 10×10-cm enhancing soft mass of the ascending colon, which was diagnosed as mucinous adenocarcinoma in a pathological report. Laboratory data showed elevated serum levels of carcinoembryonic antigen (CEA, $20.0 \mu \mathrm{g} / \mathrm{l}$; normal range, 0.0-10.0 $\mu \mathrm{g} / \mathrm{l}$ ). Certain family members of the patient had been diagnosed with endometrial, colon and pancreatic cancer. None of the family had a smoking history or presented with familial adenomatous polyposis (FAP). The patient with hereditary non-polyposis colorectal cancer (HNPCC), whose family fulfilled Amsterdam Criteria I (AC I), has remained free of disease for 15 months. Family history may be a key risk factor for MPM and HNPCC, the detailed molecular mechanisms of which remain to be elucidated. This case report with an investigation of family history may improve the clinical recognition of HNPCC and MPM.
\end{abstract}

Correspondence to: Dr Zhi-Gang Wang or Professor Qi Zheng, Department of General Surgery, The 6th People's Hospital of Shanghai, School of Medicine, Shanghai Jiaotong University, 600 Yi Shan Road, Shanghai 200233, P.R. China

E-mail: zgwang201010@yahoo.cn

E-mail: qzheng818@yahoo.cn

*Contributed equally

Key words: multiple primary malignancies, hereditary nonpolyposis colorectal cancer, family history

\section{Introduction}

Multiple primary malignancies (MPM) in a single patient are relatively rare but have increased in frequency in recent decades (1). This may be a result of medical advancements in diagnostic and therapeutic strategies, a possible effect of new carcinogens in the industrial environment and longer life span allowing another primary cancer to develop (2,3). Among those patients with MPM, double cancer is commonly observed, while triple cancers occur in $0.5 \%$ of patients and quadruple or quintuple cancers occur in less than $0.1 \%$ of patients (4). The present study describes a case of a patient with double malignancy of an undifferentiated pleomorphic sarcoma/ pleomorphic malignant fibrous histiocytoma and hereditary non-polyposis colorectal cancer (HNPCC). The literature concerning HNPCC is also discussed. Informed consent was obtained from the patient. The study was approved by the Ethics Committee of The 6th People's Hospital of Shanghai, School of Medicine, Shanghai Jiaotong University, Shanghai, China.

\section{Case report}

Patient. A 43-year-old male (proband IV12) with a past medical history of penicillin allergy and syphilis was first admitted to the hospital for a malignant histiocytoma (pG2T2bN0Mx, stage III) of the right scapula in May 2009 (Fig. 1). The patient underwent 3 tumor resections in situ due to the recurrence of the tumor in July 2009, December 2009 and January 2010. The patient was re-hospitalized in April 2010 due to a 1-month history of progressive abdominal discomfort in the right lower quadrant, increased bowel movement frequency and weight loss of $5 \mathrm{~kg}$. A computed tomography scan of the abdomen revealed an irregularly thickened wall of the ascending colon with localized stenosis and regional mesenteric lymphadenopathy (Fig. 2). Colonoscopy revealed a 10x10-cm obstructing tumor arising from the ileocecal valve to the hepatic flexure. A biopsy specimen of the tumor suggested tubulovillous adenoma with high grade dysplasia. Laboratory investigations revealed an elevated serum level of carcinoembryonic antigen (CEA, $20.0 \mu \mathrm{g} / 1$; normal range, 0.0-10.0 $\mu \mathrm{g} / \mathrm{l}$ ) and anemia with low hemoglobin $(\mathrm{Hb})$ concentration $(83.0 \mu \mathrm{g} / \mathrm{l}$; normal range, 113.0-172.0 $\mu \mathrm{g} / \mathrm{l}$ ). Carbohydrate antigen (CA) 125 and $\alpha$ fetoprotein (AFP) serum levels and other serum indices were 


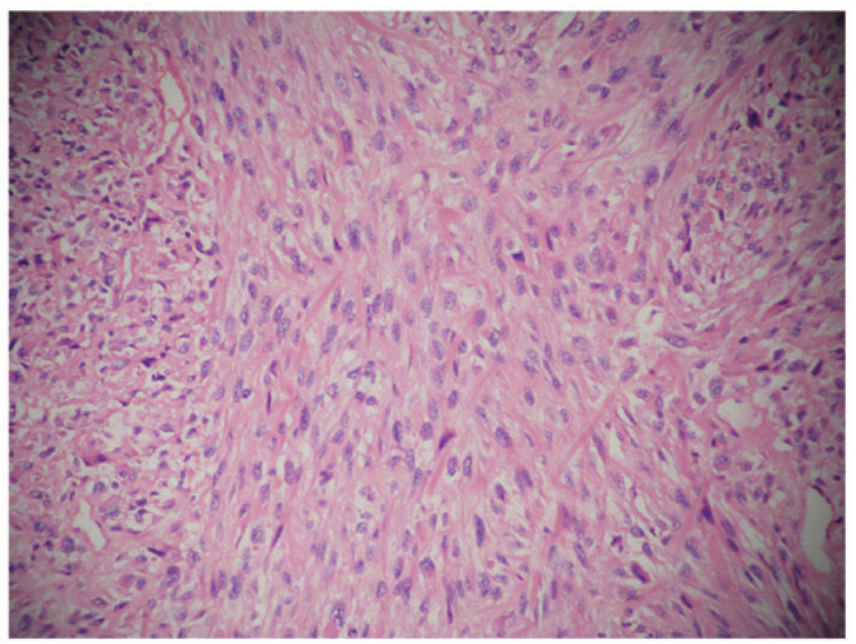

Figure 1. Microscopic findings of the right scapula. Results showed a malignant histiocytoma (H-E; original magnification, x200).

A

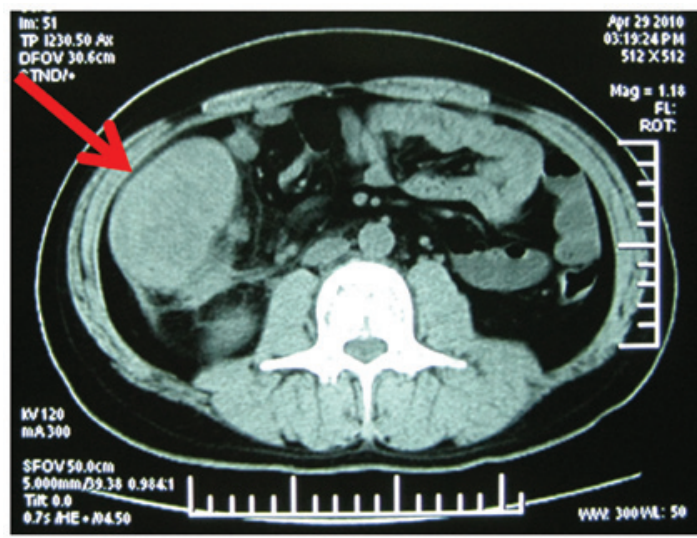

B

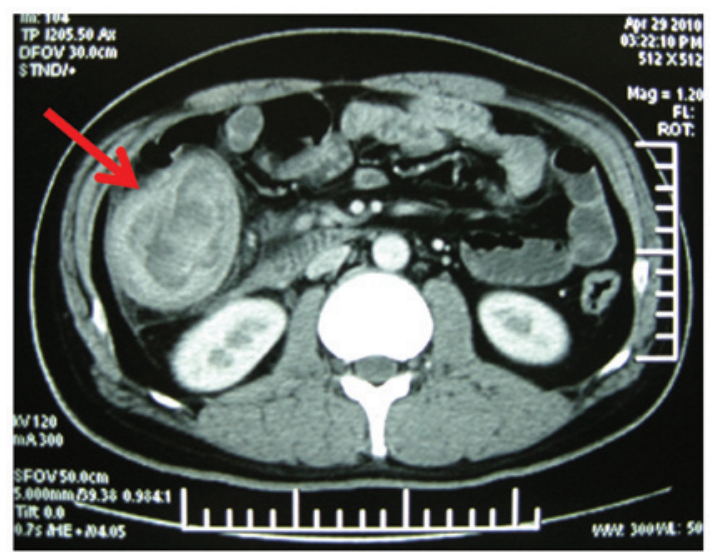

Figure 2. Preoperative computed tomography (CT) findings. (A) 10x10-cm solid mass located in ascending colon (arrows). (B) The solid mass was enhanced (arrows)

within normal limits. The patient underwent a right hemicolectomy and an end-to-end ileocolostomy. After surgery, the patient was discharged without any complications, and has been well, without any evidence of recurrence or metastasis, for 15 months with outpatient follow-up every 1-2 months. The postoperative course was uneventful, and the patient was referred for chemotherapy and radiotherapy.

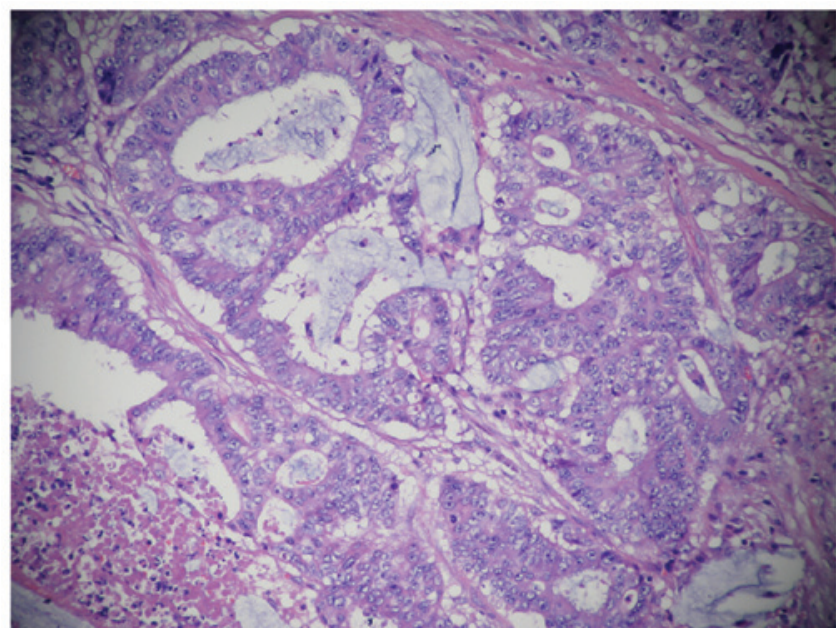

Figure 3. Microscopic findings of the colonic mass. Results showed mucinous adenocarcinoma (H-E; magnification, x200).

Pathological findings. Gross examination revealed a right hemicolectomy specimen measuring $20 \mathrm{~cm}$ in length in the terminal ileum and $30 \mathrm{~cm}$ in length in the colon. The colonic resection specimen included the cecum, ascending colon and a proximal part of the transverse colon. There was an ulcerating, circumferential mass in the cecum, $\sim 9 \times 6 \mathrm{~cm}$ in size, which extended through the entire bowel wall, infiltrating the surrounding fatty tissue. The immunohistochemical results of the tumor tissue were: $\mathrm{CK}(+), \operatorname{EMA}(+), \mathrm{P} 53(+)$, CerbB-2, EGFR(+), Ki67(60\%+), CD34 (-), S-100(-). Histologically, specimens from the tumor showed mucinous adenocarcinoma and invasion of the right renal adipose capsule, lymphatic vessel and perineural region (Fig. 3). Of the 14 peritumoral lymph nodes dissected, 1 contained metastases of the tumor. Nine mesenteric lymph nodes and 2 lymph nodes at the mesenteric root were also dissected and no metastasis was found. The immunological response of the dissected lymph nodes was $\mathrm{SH}(+), \mathrm{PH}(+), \mathrm{GH}(+)$. Specimens which were free of tumor cells exhibited chronic inflammation of the mucosa.

Family history investigation (Fig. 4). I1 succumbed to gastrointestinal cancer, the details of which could not be verified. II1 was diagnosed with colonic cancer at the age of 60 years and succumbed to the disease. III1 succumbed to colonic cancer at the age of 50 years. III2 was diagnosed with colonic cancer at the age of 70 years and succumbed to the disease 7 years after colorectomy. III 3 was diagnosed with colonic cancer at the age of 56 years and succumbed to the disease 3 years after colorectomy. III 4 was diagnosed with colonic cancer at the age of 60 years, underwent colorectomy 8 years ago and is currently well. IV1 was diagnosed with colonic cancer at the age of 30 years and succumbed to the disease 8 years after colorectomy. IV2 was diagnosed with endometrial carcinoma 8 years ago at the age of 50 years and she is currently well after surgery. IV4 was diagnosed with pancreatic cancer 2 years ago at the age of 52 years and is now well after surgery. IV6 was diagnosed with colonic cancer 4 years ago at the age of 42 years and is currently well after surgery. IV7 was diagnosed with colonic and pancreatic cancers at the age of 40 years and succumbed to the disease 


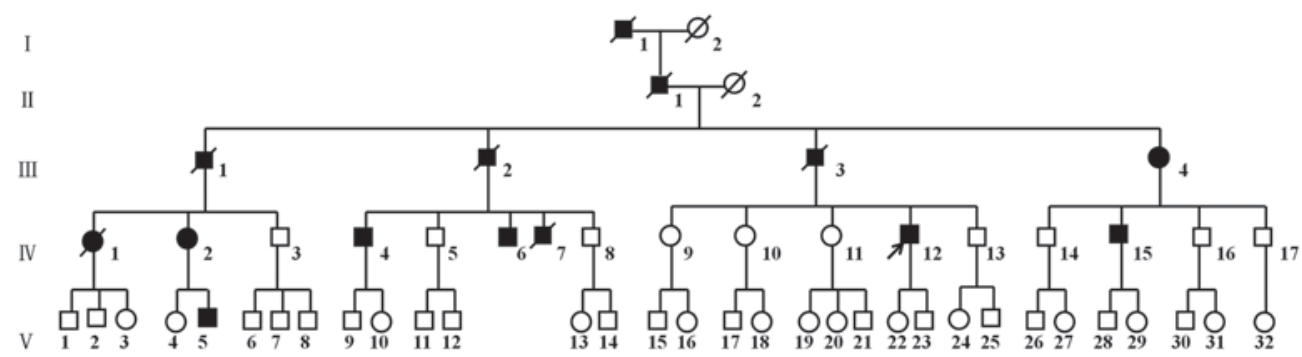

Figure 4. Family pedigrees. The patient (IV12, proband), who was diagnosed with malignant histiocytoma and colonic cancer, is indicated by an arrow. I1 succumbed to gastrointestinal cancer, the details of which could not be verified. IV2 was diagnosed with endometrial carcinoma. IV4 was diagnosed with pancreatic cancer. IV7 was diagnosed with colonic and pancreatic cancers. II1, III1, 2, 3 and 4, IV1, 6 and 15 and V5 have been diagnosed with colonic cancer.

6 years after surgery. IV12 was diagnosed with colonic cancer at the age of 41 years and is currently well after colorectomy 15 months ago. IV15 was diagnosed with colonic cancer at the age of 42 years, underwent colorectomy 1 year ago and is currently well. V5 was diagnosed with colonic cancer at the age of 32 years, underwent colorectomy 1 year ago and is currently well. IV9, 10, 11 and 13 and V22 and 23 have not been diagnosed with any cancer but have chronic gastritis, constipation and indigestion. The mean age of the patients when cancer was diagnosed in two generations (III, IV) was $56.5 \pm 12.5$ and $41.7 \pm 7.6$ years, respectively. Four generations (II, III, IV and V) underwent colonoscopy and there was no evidence of colonic polyps. There was no history of familial adenomatous polyposis (FAP) and no family spouse diagnosed with cancer.

\section{Discussion}

Ever since Billroth's report, in 1889, of a patient with multiple tumors, a gastric carcinoma that developed after the removal of a spinocellular epithelioma of the right ear, MPM have been objects of medical curiosity (5). Until 1932, only a few such cases had been recognized, when Warren and Gates classified 1,259 such patients from literature reports and post mortem examinations and modified the diagnostic criteria for MPM, which included the following $(6,7)$ : i) each tumor must present a definite picture of malignancy; ii) each tumor must be histologically distinct; and iii) the possibility that one is a metastasis of another must be excluded. A number of theories have been proposed to explain MPM but, to date, none have been proven, although the key risk factors appear to be smoking and family history (8).

In review of the literature regarding MPM, several common points may be concluded (2). First, the Japanese population appears to have a higher likelihood of developing MPM. Yamamoto et al reported that 15 to $20 \%$ of Japanese patients with colorectal carcinoma developed MPM (9). This may be caused by genetic susceptibility, longer average life span or medical advances in chemotherapy and radiotherapy. Second, most patients with MPM are geriatric. Third, smoking-related cancers, prostate cancers and renal cell carcinoma are most commonly associated with MPM (10). Fourth, head and neck cancer survivors are at an increased risk of developing another cancer of the respiratory or digestive tract (11). A 'field cancerization effect' was assumed to explain this phenomenon, with carcinogens to which the organ has been exposed initiating the proliferation of numerous clones of cells (12). Carcinogenic insults, such as tobacco and alcohol, may increase the likelihood of multiple independent malignant foci developing in the mucosa epithelium. The patient (IV12, proband) described in the present study never smoked tobacco or drank alcohol, but he received chemotherapy and radiotherapy for the first primary cancer.

The frequency of MPM depends on the length of the observation period, applied diagnostic and prognostic criteria, exposure to environmental factors, genetically defined individual susceptibility, diagnostic accuracy, followup and administered treatment. There was no family history of FAP in this case, and two family members (IV7 and IV12) have multiple malignancies. Our patient (IV12, proband) was diagnosed with malignant histiocytoma and colonic cancer, which have different histopathological criteria. Family members in this case had a high incidence of colon cancer, suggesting that certain genetic features may induce tumorgenesis.

In $15-20 \%$ of patients with colon cancer there is evidence of a family history (13). HNPCC is the most common form of hereditary colorectal cancers, with conservative estimates of $5-10 \%$ of the total colorectal cancer burden $(14,15)$. Predisposed individuals have an increased lifetime risk of developing colorectal, endometrial and other types of cancer. HNPCC may present with differing phenotypes and a detailed family history is required going back at least 3 generations (13). The first report of a cancer family that represented what is now known as HNPCC was published by Aldred Scott Warthin, a renowned pathologist, in 1913 (16). Over time, as general knowledge about HNPCC improved, more and more affected families were reported. The characteristic presentation of HNPCC is frequently right-sided localization, the presence of synchronous and metachronous colorectal carcinomas and its association with other HNPCCrelated extra-colonic tumors, including gastric, endometrial and urinary and biliary tract cancers in afflicted families (13). Pancreatic tumors have been reported in relatives with HNPCC (17). Colorectal adenocarcinoma occurring in patients with HNPCC frequently exhibits medullary features. We report a case of MPM involving tubulovillous adenoma in the ascending colon and malignant histiocytoma in the scapular with family history of colorectal carcinoma. In this family, 11 members were diagnosed with colonic cancer. The family history is notable for a high incidence of rightsided colonic cancer with no evidence of FAP. HNPCC has 
an earlier age of onset, Crohn's disease-like lymphocytic infiltration of tumor tissues, increased mucin production and a lower degree of histological differentiation compared with FAP (13). In this case report, 11 patients were male and 3 were female, and the fourth generation was markedly younger than the third generation when their cancers were first diagnosed. This family fulfills Amsterdam criteria I, with high incidence in males and early age at onset of cancer.

We are aware of some limitations in this study. First, we report only one family history investigation with the charted family tree, which limits the ability to provide robust evidence. More cases should be investigated. Second, we were unable to demonstrate a detailed explanation for the mechanism of MPM and HNPCC. Further basic research is thus needed. Despite these limitations, we believe that the present study provides evidence to improve the recognition of MPM and HNPCC, which are not well-known among practicing physicians, leading to not recognizing families at risk.

In summary, cases with MPM have been reported more often in the recent literature, but clinicians should also keep in mind that the prevalence of MPM is increasing. The assumption of a new lesion in a patient with a previous history of cancer as metastasis could possibly change the treatment strategies and delay the management of a curable neoplasm. The early detection of additional primary malignancies will enable prompt management and is likely to increase the cure rate of the disease. Multiplicity of primary malignancies itself does not necessarily indicate a poor prognosis, as long as adequate diagnosis and management are performed (2). This study suggests that family history may be a key risk factor for MPM and HNPCC, whose detailed molecular mechanism remains to be elucidated. This study, with an investigation of family history, may improve the clinical recognition of HNPCC and MPM, which is important for successful surgical treatment.

\section{Acknowledgements}

We thank Yong Hua Xu, Department of Radiology, the Central Hospital of Shanghai Xuhui District, Shanghai, China, for expert technical assistance. This research project was supported by grants from the Foundation of Shanghai Health Bureau, Shanghai, China (no. 2010L059A), and the National Natural Science Foundation of China (no. 81272401)

\section{References}

1. Youlden DR and Baade PD: The relative risk of second primary cancers in Queensland, Australia: a retrospective cohort study. BMC Cancer 11: 83, 2011.

2. Hu NC, Hsieh SC, Chen TJ and Chang JY: Multiple primary malignancies including colon, stomach, lung, breast, and liver cancer: a case report and literature review. Chin Med J (Eng) 122: 3091-3093, 2009.

3. Landgren O, Thomas A and Mailankody S: Myeloma and second primary cancers. N Engl J Med 365: 2241-2242, 2011.

4. Németh Z, Czigner J, Iván L, Ujpál M, Barabás J and Szabó G: Quadruple cancer, including triple cancers in the head and neck region. Neoplasma 49: 412-414, 2002.

5. Renda A and Carlomagno N: Nosography. In: Multiple Primary Malignancies. Andrea Renda (ed). Springer-Verlag, Italy, 2009.

6. Warren S and Gates O: Multiple primary malignant tumors: a survey of the literature and statistical study. Am J Cancer 16: 1358-1414, 1932.

7. Yamasaki M and Higuchi M: An autopsy case of synchronous quadruple cancer. Strahlentherapie 140: 275-279, 1970.

8. Angurana SL, Kapoor R, Kumar P, Khosla D, Kumar N, Sharma SC and Patel FD: Quadruple malignancy in a single patient: a case report and comprehensive review of literature. J Cancer Res Ther 6: 230-232, 2010.

9. Yamamoto S, Yoshimura K, Ri S, Fujita S, Akasu T and Moriya Y: The risk of multiple primary malignancies with colorectal carcinoma. Dis Colon Rectum 49 (10 Suppl): S30-S36, 2006.

10. Engeland A, Bjørge T, Haldorsen T and Tretli S: Use of multiple primary cancers to indicate associations between smoking and cancer incidence: an analysis of 500,000 cancer cases diagnosed in Norway during 1953-1993. Int J Cancer 70: 401-407, 1997.

11. Mussari S, Amichetti M and Tomio L: Quadruple cancer in a single patient: a report of four cases. Eur J Surg Oncol 26: 614-616, 2000.

12. Slaughter DP, Southwick HW and Smejkal W: Field cancerization in oral stratified squamous epithelium; clinical implications of multicentric origins. Cancer 6: 963-968, 1953.

13. Tanyi M, Olasz J, Kámory E, Csuka O, Tanyi JL, Ress Z and Damjanovich L: Difficulties in recognizing families with Hereditary Non-polyposis Colorectal Carcinoma.Presentation of 4 families with proven mutation. Eur J Surg Oncol 34: 1322-1327, 2008.

14. Lynch HT and Smyrk T: Hereditary nonpolyposis colorectal cancer (Lynch syndrome). An updated review. Cancer 78: 1149-1167, 1996.

15. Wagner A, Tops C, Wijnen JT, Zwinderman K, van der Meer C, Kets M, Niermeijer MF, et al: Genetic testing in hereditary nonpolyposis colorectal cancer families with a MSH2, MLH1, or MSH6 mutation. J Med Genet 39: 833-837, 2002.

16. Warthin AS: Heredity with reference to carcinoma as shown by the study of the cases examined in the pathological laboratory of the University of Michigan: 1895-1913. Arch Intern Med 12: 546-555, 1913.

17. Lynch HT, Voorhees GJ, Lanspa SJ, McGreevy PS and Lynch JF: Pancreatic carcinoma and hereditary nonpolyposis colorectal cancer: a family study. Br J Cancer 52: 271-273, 1985. 